\title{
On Generalized m-flat Modules
}

\section{Zubayda M. Ibraheem}

zubaida_almulla@yahoo.com

College of Computer Sciences and Mathematics

University of Mosul, Mosul, Iraq

Received on: 22/12/2011

Accepted on: 15/02/2012

\section{ABSTRACT}

Let $\mathrm{I}$ be a right (left) ideal of a ring $\mathrm{R}$. Then $\mathrm{R} / \mathrm{I}$ is a right (left) generalized $\mathrm{m}$ - flat modules ( $\mathrm{GmF}$ - module) if and only if for each $a \in I$, there exist $b \in I$ and a fixed positive integer $\mathrm{m}$ such that $a^{m}=b a^{m}\left(a^{m}=a^{m} b\right)$. We study characterization and properties this class of flat modules, and we give the relation between this class and generalized $\mathrm{m}$ - flat modules and $\mathrm{m}$ - regular rings, reduced rings, reversible rings and uniform rings.

Keywords: Moduls, flat, regular, reduced, reversible, uniform.

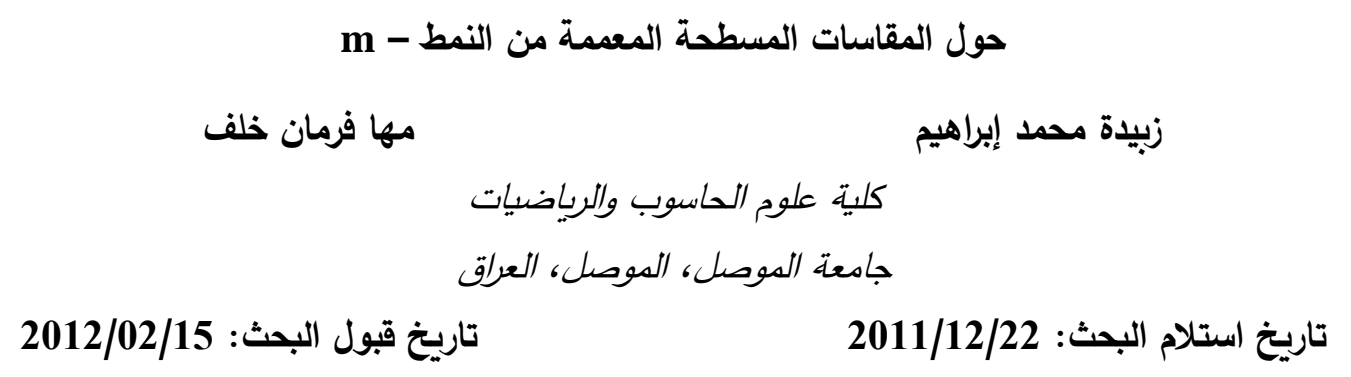

\section{الملخص}

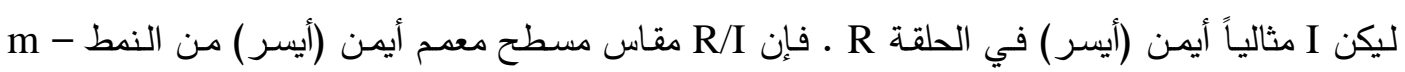

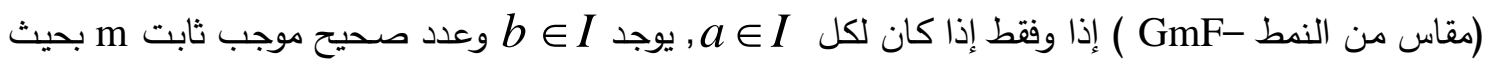
إن

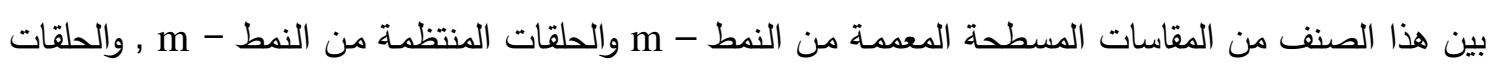
المختزلة والحلقات العكوسة والحلقات الموحدة. كلمات مفتاحية: مقاسات، مسطحة، منتظمة، مختزلة، عكوسة، موحدة. : 1

في هذا البحث R حلقة تجميعية متحايدة والمقاسـات أحادية.لكل التالف الأيمن والتالف الأيسر لـ a على التوالي , كما أثرنا بـ N ومجموعة كل العناصر المعدومة القوى على التوالي.

يقال للحلقة R بأنها حلقة موحدة (Uniform) يمنى (يسرى) إذا كان كل مثالي غير صفري أيمن (أيسر)

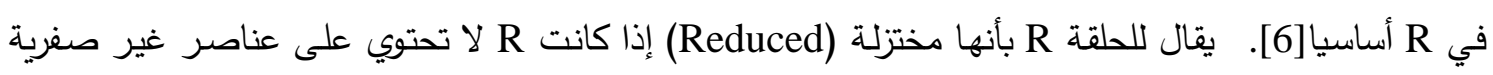
معدومـة القوى , أو بعبارة أخرى لكل عنصر عكوسة (Reversible) إذا كان 
حـلقة ديــــو بضعف اليمنى ( Weakly -duo) , إذا كان لكل

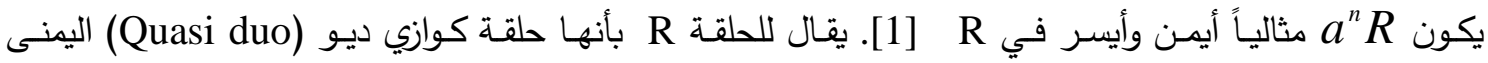

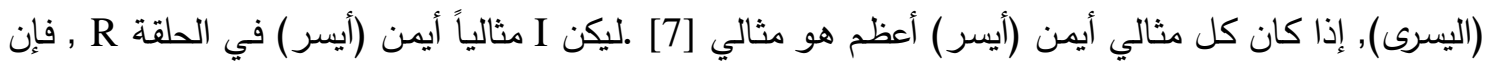

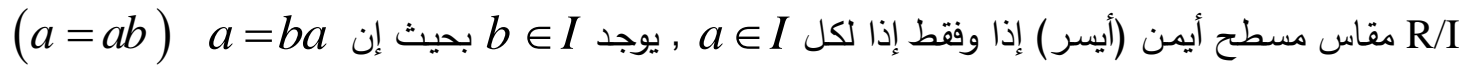
[5].يقال للحلقة R بأنها حلقة فون نتومان المنتظمة إذا كان لكل a $a \in R$ يوجد

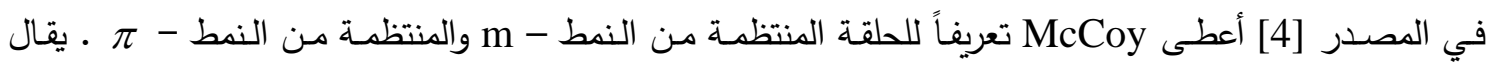

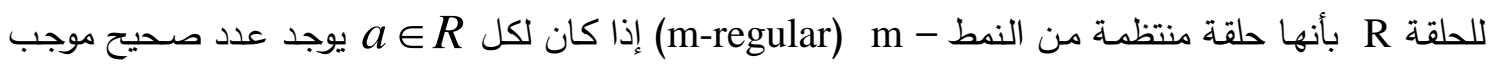
ثابت m بحيث إن R حلقة منتظمة من النمط m - 2. المقاسات المسطحة المعممة من النمط في هذا البند تم دراسة نوع من المقاسات المسطحة المعمة من النمط - m عندما تكون m عدد ثابت مع بعضاً من خواصها الأساسية. تعريف 2.1 :

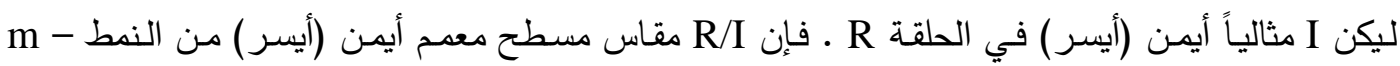

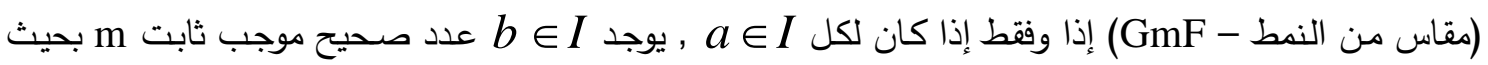
. $\left(a^{m}=a^{m} b\right) a^{m}=b a^{m}$

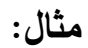

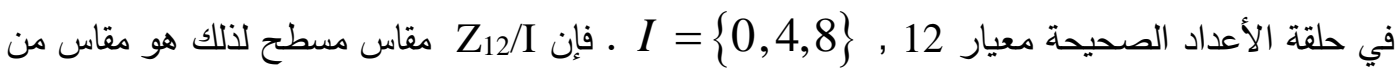
النمط - G2F.

قضية 2.2: [3] لتكن R( القضية الآتية تعطي العلاقة بين المقاسات اليمنى واليسرى من النمط - GmF. قضية 2.3: لتكن R حلقة عكوسة , وليكن R مثالياً في R/I مقاس أيسر من النمط - GmF إذا وفقط إذا كان GmF - مقاس أيمن من النمط R/I البرهان: واضح. قضية 2.4: لتكن R حلقة و M مثالياً أيمن أعظم في R , وليكن R/M مقاس أيمن من النمط - GmF. فإن كل عنصر ليس قاسماً صفرياً أيسر له معكوس أيمن. 
نفترض أن $a \neq 0$ هو عنصر ليس قاسماً صفرياً أيسر , و نفترض أن R

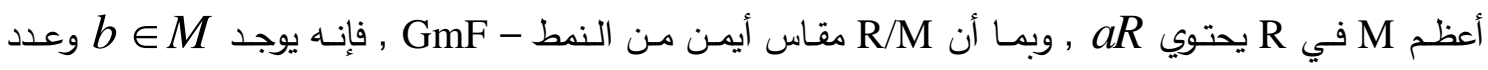
صحيح موجب ثابت m , بحيث إن

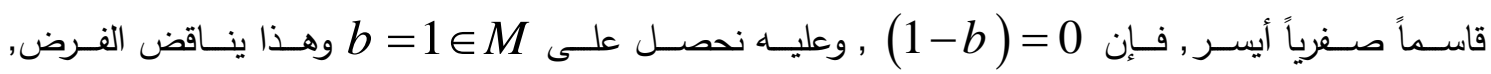

ש. إذاً [7]:2.5 قضية مساعدة إذا كانت R حلقة كوازي ديو اليمنى , فإن

القضـية الآتيـة تبـين العلاقـة بـين جذر جاكوبسون (R) J

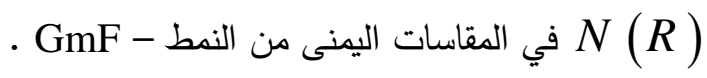

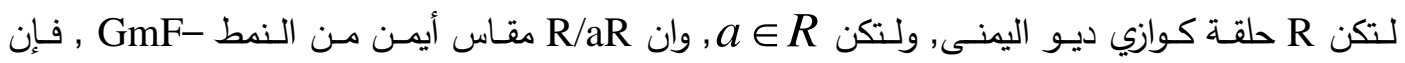

$$
. J(R)=N(R)
$$

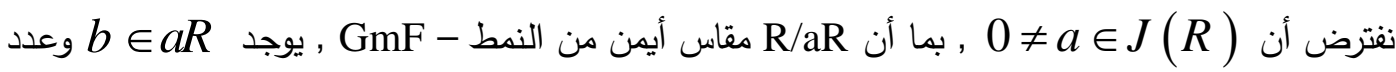
صحيح موجـب ثابـت m, بحيـث إن

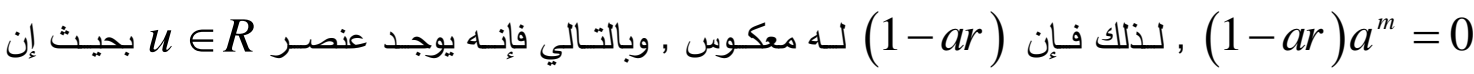
$u(1-a r)=1$

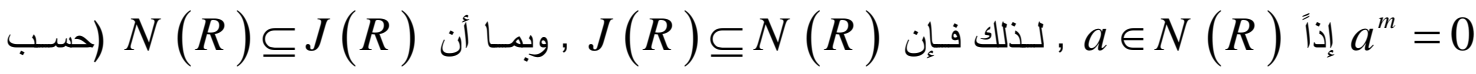
ש. القضية المساعدة 2.6) , نحصل على (R)

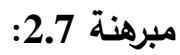

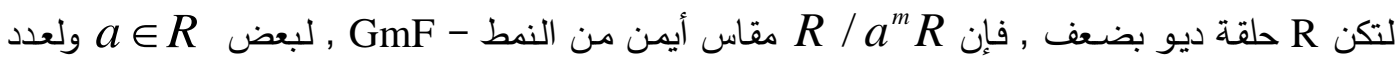

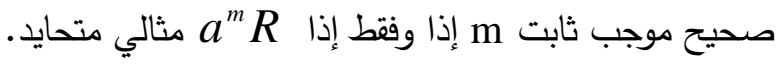
البرهان: (- n)

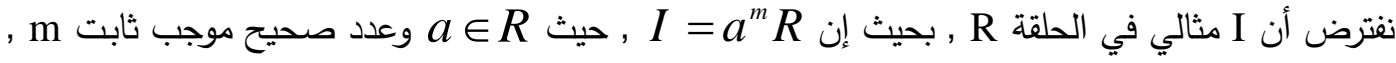
واضح أن بحيث إن $I^{2}=I$ وعليه $I \subseteq I^{2}$ 
لإلثبــــات العكـــس , نفتـــرض أن

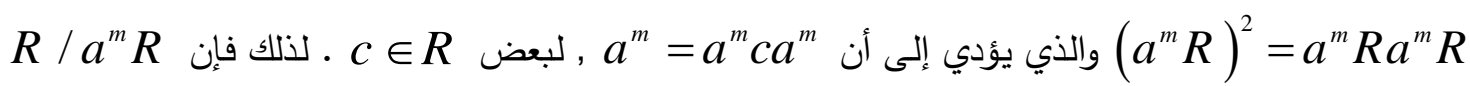

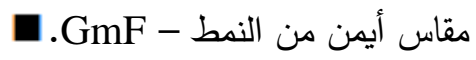
3. العلاقة بين المقاسات المسطحة من النمط - GmF والحلقات الأخرى

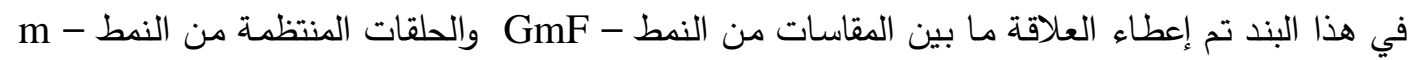
والحلقة المقسومة والحلقة الموحدة والحلقة المختزلة.

القضية الآتية تعطي العلاقة بين الحلقة الموحدة والحلقة المقسومة والمقاسات من النمط - GmF.

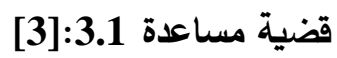
لتكن R حلقة مختزلة , فإن لكل

$$
\begin{array}{r}
. r\left(a^{n}\right)=l\left(a^{n}\right) \\
. r(a)=r\left(a^{n}\right) \\
. a^{n} R \cap r\left(a^{n}\right)=0
\end{array}
$$

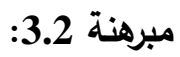

لتكن R حلقة عكوسة وموحدة يمنى M مثالياً ايمن أعظم في R , حيث R/M مقاس أيمن من النمط -

GmF

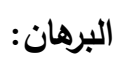

نفترض أن a

مقـاس أيمـن مـن الـنط - GmF , يوجـ

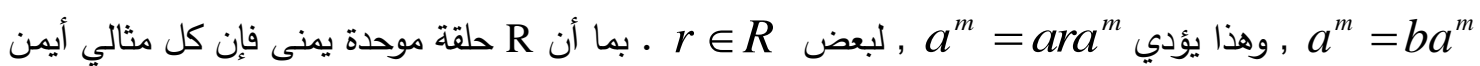
فيها يكون أساسياً . ليكن وهذا يؤدي إلى أن $a^{a r a m} z=0$ إذ

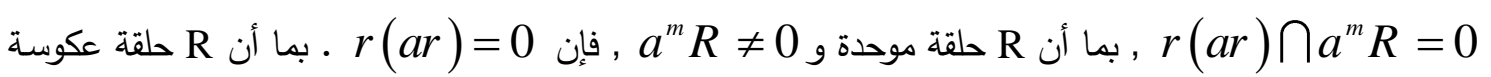

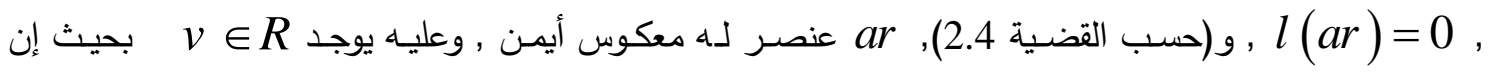

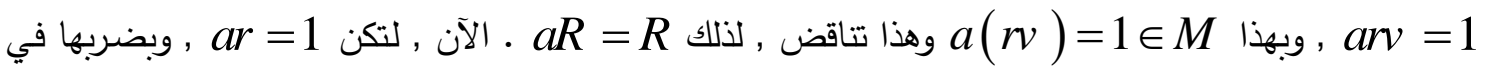

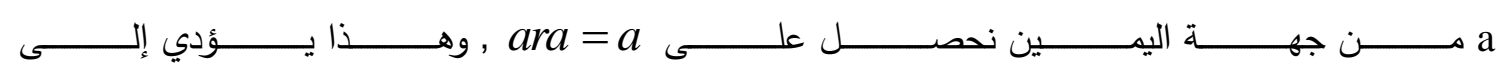

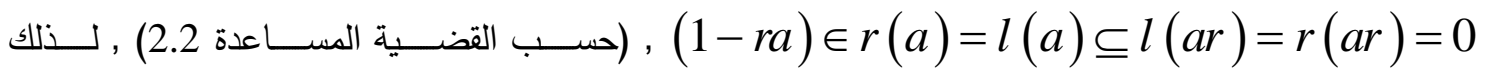

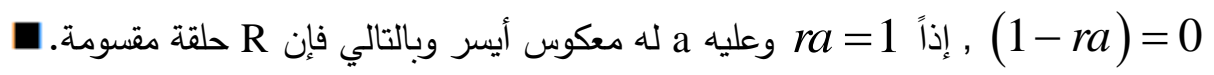


قضية 3.3:

ليكن I مثالياً في الحلقة R , إذا كانت R حلقة منتظمة من النمط - m , فإن R/I مقاس أيمن (أيسر) من

النمط - GmF. البرهان: واضح.

تعريف 3.4:

يقال للمثالي I في الحلقة R بأنه شبه أولي متمم (Completely semi-prime) إذا كان

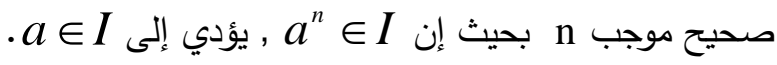

مبرهنة 3.5:

لتكن R حلقة و I مثالي شبه أولي متمم في R . فإن R/I مقاس أيسر من النمط - GmF ولكل . إذا وفقط إذا البرهان:

نفترض أن R/I مقاس أيسر من النمط - GmF , ونفترض أيضاً موجب ثابت m , بحيث إن

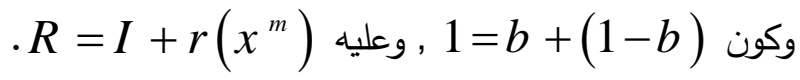
لإلثبـــات العكــس , نفتـرض أن أنس

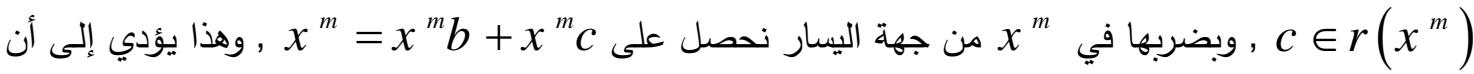

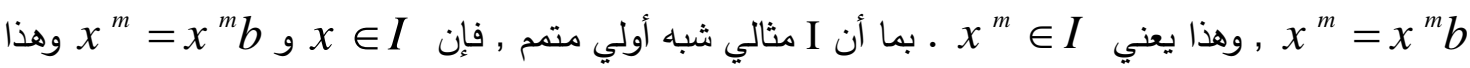

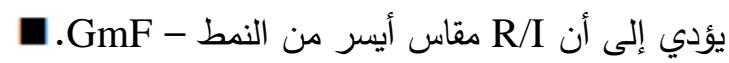
من القضية 3.3 ومبرهنة 3.5 نحصل على النتيجة الآتية. نتيجة 3.6:

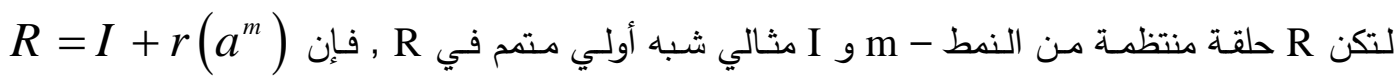
لكل a

القضية الآتية تعطي العلاقة بين الحلقات المختزلة والمقاسات اليمنى من النمط - GmF.

قضية 3.7:

لتكن R حلقة R مثالياً أيمن أعظم في R , حيث R/M مقاس أيمن من النمط - GmF فلقة R R إنة مختزلة إذا كان $l\left(a^{m}\right) \subseteq r(a)$ ولعدد صحيح موجب ثابت البرهان:

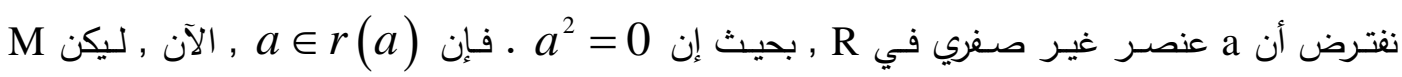
مثالياً أيمن أعظم يحتوي (a) 


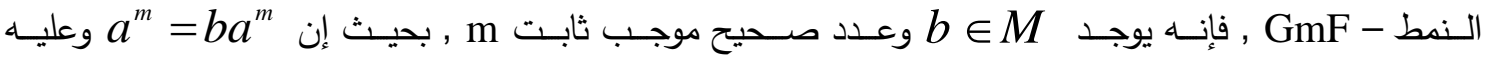
, $(1-b) \in r(a) \subset M$ إذاً , (1-b) , وهذا يؤدي إلى أن (a) ومنها ينتج $1 \in M$, وهذا تتاقض لكون قضية 3.8: لتكن R حلقة و R مثالياً أعظم حيث R/M مقاس أيمن من النمط - GmF . فإن مباشر في R. إذا كان البرهان: يجب أن نبرهن الادعاء غير صحيح يوجد مثالياً أيمن أعظم يحتوي (R/M , الآن مقاس أيمن من النمط - فـإن , GmF -

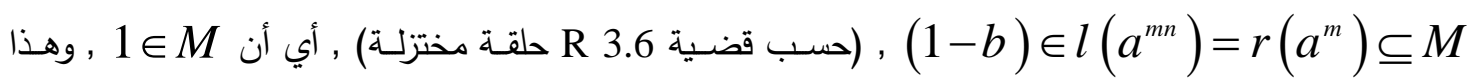
تناقض. وعليه ם.R مركبة جمع مباشر في r.1 


\section{$\underline{\text { REFERENCES }}$}

[1] Brown, S. H. (1973), Rings over which every simple module is reationally complete, Canad. J. Math. 25, PP. 693 - 701.

[2] Cohn, P. M. (1999), Reversible rings, Bull. London Math. Soc. Vol. 31, PP. $641-648$.

[3] Khalil, Sh. M. (2008), "On Generalized Pure Ideals", M. Sc. Thesis Mosul University.

[4] McCoy, N. H. (1939), Generalized regular rings , Bull. Amer. Math. Soc. Vol. 45, PP. 175 - 178.

[5] Rege, M. B. (1986), On Von Neumann regular rings and SF-rings, Math. Japonica , 31(6), PP. 927 - 936.

[6] Yue Chi Ming, R. (1985), On Von Neumann regular rings XII, Tamkang J. Math. 16 (4), PP. 67 - 75.

[7] Yu, H. P. (1995), On quasi - duo rings, Glasgow Math. J. 37 , PP. 21 - 31. 